\title{
Estado nutricional de escolares segundo a localização geográfica das escolas em Sorocaba, São Paulo
}

\author{
Nutritional status of schoolchildren according to school geographical localization in the city of Sorocaba, \\ São Paulo, Brazil
}

Cláudio Eduardo B. Martins ${ }^{1}$, Roberto Régis Ribeiro ${ }^{2}$, Antonio de Azevedo Barros Filho ${ }^{3}$

\section{RESUMO}

Objetivo: Verificar as prevalências de baixo peso, sobrepeso e obesidade em escolares, segundo a localização geográfica das escolas públicas na cidade de Sorocaba (SP), Brasil.

Métodos: Foi avaliado o maior número possível de escolares de sete a dez anos de idade, num total de 11.290 indivíduos pertencentes à rede pública municipal de escolas. Para a determinação de baixo peso, sobrepeso e obesidade foram utilizados, respectivamente, os percentis $\leq 5, \geq 85$ e $\geq 95$ do índice de massa corpórea por idade para sexo e idade propostos pelos Centers for Disease Control and Prevention (CDC, 2000). Comparou-se a prevalência de alterações nutricionais de acordo com a localização da escola.

Resultados: A prevalência de baixo peso, sobrepeso e obesidade nas crianças avaliadas, segundo a região geográfica de Sorocaba foi, respectivamente: Centro 3,4, 17,2 e 15,1\%; Sul 4,2, 15,4 e 11,2\%; Leste 3,2, 14,7 e 12,7\%; Nordeste 4,3, 11,9 e 10,7\%; Norte 5,8, 12 e 9,1\%; Noroeste 7,5, 11 e 9,1\%; Oeste 6,0, 11 e 9,2\%. Os meninos apresentaram 5,3, 12,1 e $11,9 \%$ e as meninas 5,9,12,3 e 8,2\% de baixo peso, sobrepeso e obesidade, respectivamente. Houve diferença significativa na prevalência de alterações nutricionais entre as regiões da cidade $(p<0,001)$

Conclusões: O Centro e o Leste de Sorocaba apresentaram as maiores taxas de escolares com excesso de peso, as regiões Noroeste e Oeste, as maiores taxas de baixo peso. $\mathrm{Na}$ análise por sexo, os meninos apresentaram maiores taxas de obesidade. Esses resultados podem ajudar a

Instituição: Faculdade de Ciências Médicas da Universidade Estadual de Campinas (FCM-Unicamp), Campinas, SP, Brasil

'Educador físico; Mestre em Saúde da Criança e do Adolescente; pesquisador do Centro de Investigação em Pediatria da FCM-Unicamp, Campinas, SP, Brasil

2Educador físico; Doutor em Saúde da Criança e do Adolescente; pesquisador do Centro de Investigação em Pediatria da FCM-Unicamp, Campinas, SP, Brasil

${ }^{3}$ Professor Doutor do Centro de Investigação em Pediatria da FCM-Unicamp, Campinas, SP, Brasil planejar políticas públicas para a prevenção da obesidade em escolares.

Palavras-chave: prevalência; estado nutricional; sobrepeso; obesidade; estudantes.

\section{ABSTRACT}

Objective: To estimate the prevalence of underweight, overweight and obesity in students of public schools from Sorocaba, São Paulo, Brazil.

Methods: This cross-sectional study enrolled 11,290 children aged seven to ten from public schools in the city of Sorocaba. Underweight, overweight and obesity were defined as, respectively, $\leq 5^{\text {th }}, \geq 85^{\text {th }}$ and $\geq 95^{\text {th }}$ body mass index percentiles for sex and age, according to the Centers for Disease Control and Prevention growth chart (CDC, 2000). The prevalence of nutritional alterations was assessed according to the geographical localization.

Results: Rates for underweight, overweight and obesity were respectively: Central area 3.4, 17.2 and 15.1\%; Southern area, 4.2, 15.4 and 11.2\%; Eastern area, 3.2, 14.7 and $12.7 \%$; Northeast, 4.3, 11.9 and $10.7 \%$; North, 5.8, 12 and 9.1\%; Northwest 7.5, 11 and 9.1\%; and Western regions 6, 11 and $9.2 \%$. The differences between areas were significant $(p<0.001)$. Boys had 5.3, 12.1 and $11.9 \%$ of underweight, overweight and obesity, while girls presented the rates of 5.9, 12.3 and $8.2 \%$.

Conclusions: Central and Eastern regions of the city had the highest rates of children with overweight. The northwest

Endereço para correspondência:

Antonio de Azevedo Barros Filho

Rua Tessália Vieira de Camargo, 126 - Cidade Universitária Zeferino Vaz CEP 13083-887 - Campinas/SP

E-mail: abarros@fcm.unicamp.br

Fonte financiadora: Coan Alimentação e Serviços, Habib's e Informática Wd5.

Conflito de interesse: nada a declarar

Recebido em: 23/12/2008

Aprovado em: 6/8/2009 
and western regions presented the highest underweight rates. Boys have higher rates of obesity. These results should help to plan Public Health strategies to prevent obesity in schoolchildren.

Key-words: prevalence; nutritional status; overweight; obesity; students.

\section{Introdução}

Estudos censitários nacionais do estado nutricional apontam as prevalências de macroregiões importantes para a construção de políticas públicas em nível federal e estadual. Um município necessita, além destas informações, conhecer suas particularidades para adequar programas e ações efetivos.

Projeções lineares de prevalência indicam que, por volta de 2010, cerca de 26 milhões de crianças (36\%) da Comunidade Europeia estarão com sobrepeso e 6 milhões serão obesas ${ }^{(1)}$. Com o tempo, o número previsto de crianças com sobrepeso aumentará aproximadamente 1,3 milhões ao ano e de obesas, acima de 0,3 milhões. Mais de um milhão de crianças obesas apresentarão indicadores de doenças cardiovasculares, hipertensão arterial e altos níveis de colesterol $^{(2)}$, e mais de 1,4 milhões de crianças obesas estarão em estágios iniciais de desordem do fígado ${ }^{(3)}$. A probabilidade de uma criança com sobrepeso se tornar um adulto jovem obeso aumenta dependendo do estilo de vida (sedentarismo) e da intensidade do ganho de peso.

Nos Estados Unidos, a obesidade continua a ser um problema de Saúde Pública. Segundo o National Health and Nutrition Examination Survey (NHANES) ${ }^{(4)}$, a prevalência de obesidade dobrou entre 1980 e 2004 como consequência a muitos fatores, entre eles a oferta de alimento e a diminuição das oportunidades de atividade física. O NHANES 20032004, utilizando-se de medidas de peso e altura, indicou uma estimativa de $17 \%$ de crianças e adolescentes com idade entre dois e 19 anos obesas. O sobrepeso passou de 7,2 para $13,9 \%$, em indivíduos com idade entre dois e cinco anos, e de 11 para $19 \%$ no grupo etário dos seis aos 11 anos de idade, de 1988-1994 a 2003-2004. Em adolescentes com 12 a 19 anos, o sobrepeso aumentou de 11 para $17 \%$ no mesmo período ${ }^{(4,5)}$.

No Brasil, há poucos estudos populacionais sobre prevalência do estado nutricional de crianças e adolescentes: o Estudo Nacional da Despesa Familiar (ENDEF, 1974/1975), a Pesquisa Nacional sobre Saúde e Nutrição (PNSN, 1989), a Pesquisa sobre Padrões de Vida (PPV, 1996/1997) somente nas regiões Sudeste e Nordeste do país, a Pesquisa de Orçamentos Familiares (POF, 2002/2003) e a Pesquisa Nacional de Demografia e Saúde da Criança e da Mulher (PNDS, 2006). A relevância do monitoramento do estado nutricional precoce refere-se ao processo de educação pela intensa influência ambiental e pelo fato de a obesidade ser etiologicamente multifatorial ${ }^{(6-9)}$. A prevalência de sobrepeso está em ascensão e o baixo peso tem diminuído ${ }^{(10)}$. O baixo nível socioeconômico (NSE) e seus acompanhantes intrínsecos, como más condições ambientais, culturais e familiares, são os fatores etiológicos que mais se destacam na desnutrição energético-proteica ${ }^{(11)}$. A PNDS $2006^{(10)}$ revelou situações de excesso de peso para altura em $6,6 \%$ das crianças brasileiras menores de cinco anos, indicando, neste caso, a exposição da população ao risco de obesidade na infância. A prevalência do excesso de peso para altura situou-se entre 5 e $7 \%$ na maioria dos estratos estudados, aproximando-se de 2 a $3 \%$ apenas em crianças de elevada ordem de nascimento e excedendo $8 \%$ em crianças da região Sul. Estudos de prevalência do estado nutricional de escolares, em municípios brasileiros, segundo classificação dos Centers for Disease Control and Prevention (CDC$2000)^{(12)}$, indicam alta prevalência de crianças com sobrepeso e obesidade, assim como estudos realizados em São Paulo ${ }^{(13)}$, Cosmópolis ${ }^{(14)}$, Recife ${ }^{(15)}$, Salvador ${ }^{(16)}$, Rio de Janeiro ${ }^{(17)}$, Feira de Santana ${ }^{(18)}$, Santos ${ }^{(19)}$ e Capão da Canoa ${ }^{(20)}$.

Com o objetivo de conhecer a relação entre estado nutricional e localização geográfica da escola na zona urbana da cidade de Sorocaba (SP), o presente estudo descreveu e comparou, por meio do índice de massa corpórea (IMC), as prevalências de baixo peso, sobrepeso e obesidade de alunos de escolas públicas municipais, de acordo com sexo, idade e região geográfica da escola, segundo os critérios dos CDC 2000 .

\section{Métodos}

O estudo foi descritivo, com delineamento transversal, realizado na área urbana da cidade de Sorocaba, localizada na região sudoeste do Estado de São Paulo, com estimativa de 597.683 habitantes em 2008 e taxa de urbanização de 98,88\% em 2007. Como indicadores de saúde, a taxa de natalidade foi estimada em 13,72 (por mil habitantes), nascimentos de baixo peso em 8,07\% (menos de 2,5kg) e 8,57\% de gestações pré-termo em 2006; mortalidade infantil de 13,49 por mil nascidos vivos e mortalidade na infância de 17,57 por mil nascidos vivos em 2007. Um total de 96,59\% dos domicílios 
possuem infraestrutura interna urbana adequada. É a quinta cidade em desenvolvimento econômico do Estado, com investimentos perto de 3,5 bilhões de dólares e PIB avaliado em 9 bilhões de reais, em 2005, o que representa cerca de 0,5\% do PIB do país e 1,5\% de São Paulo. A renda per capita média foi de 2,95 salários mínimos, com 10,6\% de pobres (renda domiciliar per capita inferior a 0,5 salário mínimo) em 2000, e desigualdade social, medida pelo Índice de Gini, de 0,55. A educação é classificada pelo Índice de Educação (IDHM-E) com 0,915 pontos. As condições de vida foram classificadas em 2004, pelo Índice Paulista de Responsabilidade Social (IPRS), no Grupo 1 (municípios com nível elevado de riqueza e bons níveis nos indicadores sociais); apresenta Índice de Desenvolvimento Humano Municipal (IDH-M) de 0,828, ocupando a $38^{a}$ colocação no ranking Estadual e a $145^{\text {a }}$ posição no ranking $\mathrm{Nacional}^{(21-23)}$.

Foram objetos de estudo os escolares de sete a dez anos de idade, da primeira à quarta série do Ensino Fundamental, em 27 escolas da rede pública municipal, distribuídas em regiões geográficas: centro, leste, nordeste, norte, noroeste, oeste e sul. Tais regiões foram delimitadas por posição geográfica, vias de acesso e contingente populacional e discriminadas por meio de dados sociais e de infraestrutura urbana (Figura 1) ${ }^{(24,25)}$. As regiões Norte e Noroeste possuem bairros em situação de pobreza e contrastes sociais, principalmente na periferia (região suburbana), em decorrência de grande expansão imobiliária. Em sua periferia situa-se o Habiteto, projeto de transfavelamento que abriga famílias em situação de miséria ${ }^{(25)}$. A região Oeste, em processo de expansão territorial, apresenta contrastes entre os bairros próximos e suburbanos, carentes de infraestrutura, com famílias em situação de miséria como o Jardim Ipiranga e o Nova Esperança ${ }^{(25)}$. Na região Nordeste, localiza-se a "zona industrial”, com dois bairros populosos e suburbanos, porém com menor taxa de pobreza e melhor infraestrutura $^{(24)}$. A região sul apresenta a maior concentração de riqueza da cidade e se configura como um dos principais locais de investimento imobiliário e empresarial. A região Leste é uma das mais antigas e, historicamente, foi sede de várias indústrias têxteis, com predomínio de classe média e pouca pobreza ${ }^{(25)}$. O centro é delimitado por quatro vias principais, incluindo os bairros circunvizinhos, com baixíssima taxa de pobreza ${ }^{(24)}$.

O estudo teve apoio público municipal mediante assinatura do termo de consentimento livre e esclarecido pelo prefeito, pela secretária da educação, pelos diretores de cada escola municipal participante, posteriormente encaminhado aos pais e responsáveis. A realização do estudo obedeceu aos princípios éticos para pesquisa envolvendo seres humanos, conforme resolução CNS 196/96, e foi aprovada pelo Comitê de Ética em Pesquisa da Faculdade de Ciências Médicas da Universidade Estadual de Campinas.

Os critérios de inclusão para as crianças foram: estar regularmente matriculada nas escolas públicas municipais avaliadas; ter entre sete e dez anos de idade, sendo considerada a idade decimal. A caracterização do baixo peso, sobrepeso ou obesidade seguiu os critérios dos CDC, 2000. Os critérios de exclusão foram: escolares que não estivessem aptos à realização da antropometria no momento da coleta de dados; portadores de necessidades especiais e meninas que tiveram menarca. Foi avaliado o maior número possível de alunos aptos dentre 20.450 matriculados na rede municipal de um total de 82.150 matriculados nesta faixa etária em todo o município ${ }^{(26)}$.

Diretores, professores de educação física e estagiários das escolas participaram de palestras-treinamento a respeito do projeto, vivenciando teoria e prática da metodologia utilizada. As crianças presentes em horário de aula passaram por medidas de massa corporal (peso) e estatura, realizadas pelos professores de educação física e estagiários das escolas, nas datas estipuladas para as avaliações, previamente agendadas com a direção de cada escola, durante o período de fevereiro a agosto de 2006.

As medidas foram executadas utilizando-se de padronização universal por meio de estadiômetros portáteis da

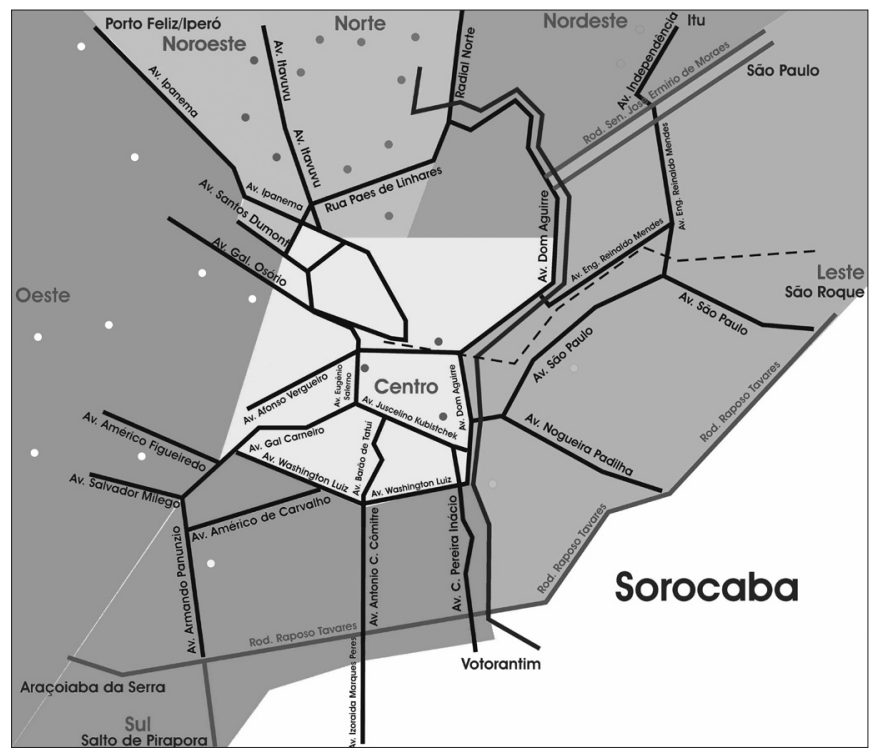

Figura 1 - Mapa geográfico de Sorocaba com divisão por regiões. Os pontos em negrito representam as escolas municipais avaliadas. 
marca WCS ${ }^{\circledR}$ e balanças portáteis Tanita ${ }^{\circledR}$ BF682. Também foi calculado o IMC dos participantes. Foram consideradas de baixo peso as crianças com IMC para a idade menor ou igual ao percentil 5; com sobrepeso, as crianças com IMC para a idade maior ou igual ao percentil 85 e menor que o percentil 95 e, com obesidade, as crianças com o IMC para a idade maior ou igual ao percentil 95 .

Os dados foram inseridos e armazenados, por meio de dupla digitação, em programa computacional. As análises estatísticas foram conduzidas utilizando-se o software Statistical Package for the Social Sciences (SPSS), versão 13.0. Foi realizada análise univariada, para verificar a prevalência de cada uma das variáveis estudadas, peso, estatura e IMC, com significância se $p<0,05$. O teste do qui-quadrado foi aplicado para comparar as variáveis não-paramétricas. Para identificar diferenças entre as prevalências encontradas no IMC do desfecho (baixo peso, sobrepeso e obesidade) nos diferentes grupos, usou-se o teste de Tukey para comparações múltiplas ${ }^{(27)}$.

\section{Resultados}

Foram avaliados 5.669 meninos $(50,2 \%)$ com média

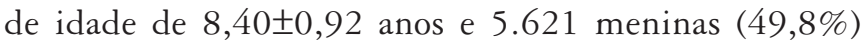
com média de idade de 8,4 $\pm 0,9$ anos em 27 escolas (Ta- bela 1). A abrangência do estudo foi de $55,2 \%$ do total de alunos matriculados nas escolas públicas municipais e de $13,7 \%$ da população escolar de Sorocaba no Ensino Fundamental.

A análise descritiva do IMC por sexo e idade é apresentada na Tabela 2. A associação das médias do IMC entre os sexos apresentou diferenças significativas $(p<0,001)$ em cada classificação do estado nutricional. A associação entre as médias do IMC de acordo com o sexo e a idade encontrou diferenças significantes aos sete anos $(p<0,001)$, aos nove anos $(p<0,001)$ e aos dez anos $(p=0,002)$.

A Tabela 3 mostra a comparação das médias do IMC entre as regiões. A Tabela 4 apresenta a comparação do valor $Z$ do IMC entre as regiões. Analisando-se o valor $Z$ do IMC para idade, verificou-se diferença positiva apenas entre os sete e os nove anos $(p=0,001)$.

A distribuição do estado nutricional por sexo e regiões encontra-se na Tabela 5. A análise do valor $\mathrm{Z}$ da estatura para idade mostrou que 1,7\% dos escolares (187 casos, 109 meninas e 78 meninos) se encontraram abaixo de $-2 \mathrm{Z}$, sendo que 69 deles residiam na região Oeste $(0,6 \%)$ e $57(0,5 \%)$ na região Norte. Abaixo de $-3 \mathrm{Z}$ encontrou-se $0,2 \%$ dos escolares (16 meninas e cinco meninos), dos quais sete $(0,06 \%)$ residiam na região Nordeste, cinco $(0,04 \%)$ na região Norte e quatro $(0,03 \%)$ na região Oeste.

Tabela 1 - Distribuição absoluta e relativa do número de escolas municipais existentes e avaliadas e alunos matriculados e avaliados, por região geográfica na cidade de Sorocaba (SP)

\begin{tabular}{lccrccc}
\hline \multirow{2}{*}{ Regiões } & \multicolumn{3}{c}{ Escolas } & \multicolumn{3}{c}{ Alunos } \\
\cline { 2 - 7 } & Avaliadas & Existentes & $\%$ & Avaliados & Matriculados & $\%$ \\
\hline Centro & 3 & 3 & 100,0 & 958 & 1.295 & 74,0 \\
Leste & 2 & 3 & 66,7 & 708 & 1.399 & 50,3 \\
Nordeste & 2 & 2 & 100,0 & 932 & 1.394 & 66,9 \\
Noroeste & 3 & 4 & 75,0 & 1.543 & 3.019 & 51,1 \\
Norte & 8 & 11 & 72,7 & 3.174 & 7.494 & 42,2 \\
Oeste & 8 & 8 & 100,0 & 3.761 & 5.289 & 71,1 \\
Sul & 1 & 1 & 100,0 & 214 & 560 & 38,2 \\
Total & 27 & 32 & 84,4 & 11.290 & 20.450 & 55,2 \\
\hline
\end{tabular}

Tabela 2 - Distribuição dos participantes segundo sexo, idade, valores médios, mínimo, máximo e desvio padrão de IMC (kg/m²)

\begin{tabular}{|c|c|c|c|c|c|c|c|}
\hline \multirow{3}{*}{$\begin{array}{l}\text { Idade } \\
\text { (anos) }\end{array}$} & \multicolumn{3}{|c|}{ Masculino } & \multicolumn{3}{|c|}{ Feminino } & \multirow{3}{*}{ Tota } \\
\hline & \multirow{2}{*}{$\mathbf{n}$} & IMC & \multirow{2}{*}{ Mín. e Máx. } & \multirow{2}{*}{$\mathbf{n}$} & IMC & \multirow{2}{*}{ Mín. e Máx. } & \\
\hline & & $\mathrm{X} \pm \mathrm{DP}$ & & & $\mathrm{X} \pm \mathrm{DP}$ & & \\
\hline 7 & 1.233 & $16,6 \pm 2,5$ & $11,3-29,0$ & 1.226 & $16,3 \pm 2,3$ & $11,2-31,4$ & 2.459 \\
\hline 8 & 1.777 & $16,8 \pm 2,8$ & $10,9-33,4$ & 1.782 & $16,8 \pm 2,8$ & $10,1-31,0$ & 3.559 \\
\hline 9 & 1.846 & $17,4 \pm 3,1$ & $10,1-33,7$ & 1.779 & $17,1 \pm 3,0$ & $11,2-32,4$ & 3.625 \\
\hline 10 & 813 & $17,9 \pm 3,5$ & $12,4-49,3$ & 834 & $17,5 \pm 3,1$ & $12,0-34,6$ & 1.647 \\
\hline Total & 5.669 & $17,1 \pm 3,0$ & $10,1-49,3$ & 5.621 & $16,8 \pm 2,8$ & $10,1-34,6$ & 11.290 \\
\hline
\end{tabular}

DP: desvio padrão. 
Tabela 3 - Comparação multivariada do IMC por idade entre as regiões da cidade de Sorocaba (SP)

\begin{tabular}{|c|c|c|c|c|}
\hline & & Centro & Leste & Nordeste \\
\hline \multirow[t]{3}{*}{ Nordeste } & $\neq$ médias & $-0,59$ & & \\
\hline & Significância & $<0,001$ & & \\
\hline & Intervalo de confiança 95\% & $0,20-0,99$ & & \\
\hline \multirow[t]{3}{*}{ Noroeste } & $\neq$ médias & $-0,97$ & $-0,62$ & $-0,37$ \\
\hline & Significância & $<0,001$ & $<0,001$ & 0,03 \\
\hline & Intervalo de confiança $95 \%$ & $0,62-1,32$ & $0,24-1,01$ & $0,02-0,72$ \\
\hline \multirow[t]{3}{*}{ Norte } & $\neq$ médias & $-0,86$ & $-0,52$ & \\
\hline & Significância & $<0,001$ & $<0,001$ & \\
\hline & Intervalo de confiança $95 \%$ & $0,55-1,18$ & $0,17-0,87$ & \\
\hline \multirow[t]{3}{*}{ Oeste } & $\neq$ médias & $-0,93$ & $-0,59$ & $-0,34$ \\
\hline & Significância & $<0,001$ & $<0,001$ & 0,02 \\
\hline & Intervalo de confiança 95\% & $0,62-1,24$ & $0,24-0,94$ & $0,02-0,65$ \\
\hline
\end{tabular}

Tabela 4 - Comparação multivariada do valor Z do IMC por idade, entre as regiões da cidade de Sorocaba (SP)

\begin{tabular}{|c|c|c|c|c|c|}
\hline & & Centro & Leste & Nordeste & Noroeste \\
\hline \multirow[t]{3}{*}{ Nordeste } & $\neq$ médias & $-0,17$ & & & \\
\hline & Significância & 0,02 & & & \\
\hline & Intervalo de confiança $95 \%$ & $0,02-0,32$ & & & \\
\hline \multirow[t]{3}{*}{ Noroeste } & $\neq$ médias & $-0,38$ & $-0,28$ & $-0,21$ & \\
\hline & Significância & $<0,001$ & $<0,001$ & $<0,001$ & \\
\hline & Intervalo de confiança $95 \%$ & $0,24-0,52$ & $0,13-0,43$ & $0,07-0,34$ & \\
\hline \multirow[t]{3}{*}{ Norte } & $\neq$ médias & $-0,31$ & $-0,22$ & $-0,14$ & \\
\hline & Significância & $<0,001$ & $<0,001$ & 0,01 & \\
\hline & Intervalo de confiança $95 \%$ & $0,19-0,44$ & $0,08-0,35$ & $0,02-0,27$ & \\
\hline \multirow[t]{3}{*}{ Oeste } & $\neq$ médias & $-0,33$ & $-0,23$ & $-0,16$ & \\
\hline & Significância & $<0,001$ & $<0,001$ & $<0,001$ & \\
\hline & Intervalo de confiança $95 \%$ & $0,21-0,45$ & $0,10-0,37$ & $0,04-0,28$ & \\
\hline \multirow[t]{3}{*}{ Sul } & $\neq$ médias & & & & 0,28 \\
\hline & Significância & & & & 0,01 \\
\hline & Intervalo de confiança 95\% & & & & $-0,52$ a $-0,03$ \\
\hline
\end{tabular}

Tabela 5 - Distribuição de frequência absoluta e relativa, por sexo e região geográfica da cidade de Sorocaba (SP), segundo classificação do *CDC

\begin{tabular}{|c|c|c|c|c|c|c|c|c|c|c|c|c|c|c|c|}
\hline \multirow{2}{*}{ Região } & \multicolumn{3}{|c|}{ Total } & \multicolumn{4}{|c|}{ Baixo peso } & \multicolumn{4}{|c|}{ Sobrepeso } & \multicolumn{4}{|c|}{ Obesidade } \\
\hline & $\mathrm{n}$ & $\hat{0}$ & $q$ & $\hat{0}$ & $\%$ & $q$ & $\%$ & 0 & $\%$ & $q$ & $\%$ & $\hat{0}$ & $\%$ & q & $\%$ \\
\hline Centro & 958 & 464 & 494 & 13 & 2,8 & 20 & 4,0 & 77 & 16,6 & 88 & 17,8 & 92 & 19,8 & 53 & 10,7 \\
\hline Leste & 708 & 378 & 330 & 8 & 2,1 & 15 & 4,5 & 45 & 11,9 & 59 & 17,9 & 56 & 14,8 & 34 & 10,3 \\
\hline Nordeste & 932 & 476 & 456 & 22 & 4,6 & 18 & 3,9 & 54 & 11,3 & 57 & 12,5 & 63 & 13,2 & 37 & 8,1 \\
\hline Noroeste & 1.543 & 801 & 742 & 58 & 7,2 & 58 & 7,8 & 92 & 11,5 & 78 & 10,5 & 81 & 10,1 & 60 & 8,1 \\
\hline Norte & 3.174 & 1.562 & 1.612 & 91 & 5,8 & 94 & 5,8 & 187 & 12,0 & 193 & 12,0 & 162 & 10,4 & 127 & 7,9 \\
\hline Oeste & 3.761 & 1.876 & 1.885 & 105 & 5,6 & 120 & 6,4 & 209 & 11,1 & 205 & 10,9 & 202 & 10,8 & 145 & 7,7 \\
\hline Sul & 214 & 112 & 102 & 3 & 2,7 & 6 & 5,9 & 20 & 17,9 & 13 & 12,7 & 17 & 15,2 & 7 & 6,9 \\
\hline Total & 11.290 & 5.669 & 5.621 & 300 & 5,3 & 331 & 5,9 & 684 & 12,1 & 693 & 12,3 & 673 & 11,9 & 463 & 8,2 \\
\hline
\end{tabular}




\section{Discussão}

Parte dos problemas de saúde e nutrição infantis está relacionada ao padrão de vida da população, incluindo o acesso à alimentação, educação, moradia, assistência à saúde, saneamento básico, transporte, lazer e atividades físicas ${ }^{(28)}$. Crianças que residem em diferentes condições de urbanização e nível socioeconômico recebem a influência do seu meio. Estudos étnico-raciais norte-americanos em crianças e adolescentes, categorizadas por NSE e nível de urbanização (área urbana e rural), são complexos e têm pouca consistência ${ }^{(23)}$. Dados recentes do NHANES ${ }^{(4)}$ mostram associação inversa entre a prevalência de obesidade e o NSE (observado em meninas brancas) e, ao mesmo tempo, alto NSE e obesidade (em meninas afro-americanas). Variações geográficas de obesidade foram reladas nos estados americanos, acompanhando o nível de urbanização. De acordo com o National Health Interview Survey (NHIS), alta prevalência de obesidade foi encontrada nas populações rurais em comparação às urbanas e suburbanas ${ }^{(29)}$.

Sorocaba, apesar dos bons índices de desenvolvimento ${ }^{(21-}$ ${ }^{23)}$, possui problemas relacionados ao crescimento urbano e ao paralelo crescimento das periferias ${ }^{(24)}$, o que aponta uma diferenciação de condições sociais. Estudo denominado "Mapa da Fome"(25), realizado por 82 organizações não-governamentais em Sorocaba em 2003, revela que $8,7 \%$ do total da população da cidade vivia em situação de miséria, sendo mais afetados os bairros da zona Norte e Oeste. Porém, havia miseráveis em outros 197 bairros. Quase metade dessa população é composta por crianças e adolescentes.

O diagnóstico do estado nutricional dos escolares por localização geográfica das escolas pode ser uma importante ferramenta para elaborar políticas públicas de prevenção e controle da obesidade e baixo peso infantis em Sorocaba. Embora o presente estudo tenha uma limitação amostral e carência na caracterização das regiões, os achados são relevantes. Segundo a classificação dos CDC e baseando-se no IMC para a idade, a população escolar urbana pesquisada apresentou, em média, 12,2 e 10,1\% de sobrepeso e obesidade, respectivamente. Oliveira et al ${ }^{(18)}$, estudando 699 crianças de escolas públicas em Feira de Santana (BA), encontraram $6,5 \%$ de sobrepeso e $2,7 \%$ de obesidade. Estudo realizado com 7.983 escolares entre sete e dez anos da rede pública e privada da cidade de Santos, Costa $e$ a $a^{(19)}$ diagnosticaram $15,7 \%$ de sobrepeso e $18 \%$ de obesidade. Em Salvador, Leão et al ${ }^{(16)}$, avaliando 387 crianças, encontraram $15,8 \%$ de obesidade, sendo $8,2 \%$ nas escolas públicas. Marins et al ${ }^{(30)}$, em estudo de base domiciliar com 493 crianças de seis a 11 anos de idade do Rio de Janeiro, constataram uma taxa de $37,1 \%$ com excesso de peso. Suñé et al ${ }^{(20)}$, ao avaliarem 452 escolares entre 11 e 13 anos da rede pública municipal de Capão da Canoa (RS), encontraram 25,7\% de excesso de peso. Em Sorocaba observou-se prevalência de 22,3\% para essa condição.

A menor prevalência de excesso de peso em Sorocaba foi verificada no sexo feminino $(20,6 \%)$, resultado inferior aos encontrados em Santos e no Rio de Janeiro, respectivamente 32,1 e $36,4 \%$. No sexo masculino, Sorocaba apresentou 23,9\% de excesso de peso, valores menores que Santos $(30,5 \%)$ e Rio de Janeiro $(37,8 \%)$. Para $p \geq 95$, no Rio de Janeiro, Marins et al ${ }^{(30)}$ encontraram 26,4\% em meninos e $30,3 \%$ em meninas. Em Santos ${ }^{(19)}$, os meninos da rede pública tiveram maior prevalência de obesidade em relação às meninas, 16,8 versus $14,2 \%$, respectivamente. Tais valores foram mais altos do que os encontrados em Sorocaba: 11,9 e $8,2 \%$ para o sexo masculino e feminino, respectivamente. Anjos $e t a^{(17)}$, em estudo realizado com amostra probabilística de escolares de até dez anos de idade da rede pública do Rio de Janeiro, calcularam o valor $Z$ do IMC para idade e estatura para idade em comparação às curvas do NCHS (National Center for Health Statistics) e encontraram 16,8 e 16,9\% de sobrepeso em meninas e meninos, respectivamente, sendo $5,7 \%$ das meninas e $5,1 \%$ dos meninos obesos.

Analisando-se o IMC entre as regiões, o centro foi significativamente diferente, não sendo diferente apenas da região leste. A região central apresentou a maior frequência de indivíduos com sobrepeso e obesidade: 17,2 e $15,1 \%$, respectivamente, totalizando $32,3 \%$ de crianças $\operatorname{com} p \geq 85$, dos quais $36,4 \%$ eram meninos e $28,5 \%$ meninas. Valores próximos aos do centro de Sorocaba foram encontrados na cidade de Santos ${ }^{(19)}$ ( $35,1 \%$ nos meninos) e em Salvador ${ }^{(16)}$ (30\% de obesos dentre 387 crianças). Os resultados obtidos no centro de Sorocaba foram maiores do que os notados por Oliveira et a $l^{(18)}$ em escolas particulares de Feira de Santana (13,4\% de sobrepeso e 7,0\% de obesidade), mas menores do que os de Balaban e Silva ${ }^{(15)}$ em uma única escola particular da cidade de Recife (332 avaliados), onde o sobrepeso foi de $33,6 \%$ nos meninos e $35,0 \%$ nas meninas.

A região Leste foi a segunda maior quanto à prevalência de sobrepeso e obesidade (14,7 e 12,7\%, respectivamente), 
com 27,4\% de indivíduos $\operatorname{com} p \geq 85$, valores similares aos encontrados em Capão da Canoa (RS) $(25,7 \%)^{(20)}$. A região Sul, com 26,6\% de crianças com $p \geq 85$ (33\% meninos e 19,6\% de meninas) foi a terceira maior com excesso de peso, havendo apenas uma escola nessa região. As regiões Noroeste (11 e 9,1\%), Oeste (11 e 9,2\%), Norte (12 e 9,1\%) e Nordeste $(10,7$ e $11,9 \%)$ se assemelharam quanto aos níveis de sobrepeso e obesidade. Embora elevadas, estas regiões apresentaram as menores taxas de obesidade.

Os resultados encontrados em Sorocaba apontaram o centro, seguido das regiões Leste e Sul, como regiões com excesso de peso, dados que corroboram a hipótese de que vem ocorrendo um aumento do excesso de gordura corporal em crianças brasileiras ${ }^{(1,15-20)}$, na América do Norte $^{(4,5)}$ e na Europa ${ }^{(1,2)}$. Os valores obtidos na presente pesquisa são bastante elevados comparando-se aos resultados da PPV1996/1997 (17,4\%). Há uma elevada prevalência média de sobrepeso e obesidade nos escolares da primeira à quarta série, desde os sete anos.

A região Noroeste apresentou a maior taxa de baixo peso do município ( $7,2 \%$ meninos e $7,8 \%$ meninas). A região Oeste apresentou $6,0 \%$ de baixo peso $(5,6 \%$ meninos e $6,4 \%$ meninas). Tais achados podem, mais uma vez, resultar de diferenças de NSE e de fatores ambientais. Inversamente proporcional às altas prevalências de excesso de peso, registraram-se menores taxas de baixo peso na região Leste,
$(3,2 \%)$, no centro $(3,4 \%)$ e na zona Sul $(4,2 \%)$. Invariavelmente, as prevalências de baixo peso nos meninos foram inferiores às das meninas.

Em conclusão, verificou-se diferença na prevalência de baixo peso, sobrepeso e obesidade em escolares de acordo com a localização geográfica das escolas, na cidade de Sorocaba (SP). As diferenças de NSE e de fatores ambientais entre o centro e a periferia podem ser determinantes e ainda devem ser pesquisados. Programas específicos e contínuos de educação alimentar nas escolas, estímulo aos hábitos familiares de prática de exercício físico, fomento de programas de exercício físico escolares, valorizando e priorizando a educação física e o encorajamento à prática de atividade física diária, como caminhadas e o ciclismo, devem ser priorizados para evitar, na infância e adolescência, o aparecimento de doenças relacionadas ao excesso de peso.

\section{Agradecimentos}

Ao Dr. Vitor Lippi, prefeito municipal, à equipe da Secretaria da Educação e a todos os professores e estagiários de educação física, pelo valioso auxílio na coleta de dados. Aos Srs. Nilson Dimas, Alessandro Santos, Ariel Mendes, Patrícia Arjona e Allana Bacci, pelo suporte e companheirismo. Finalmente, aos nossos patrocinadores Coan Alimentação e Serviços, Habib’s e Wd5 informática.

\section{Referências bibliográficas}

1. Jackson-Leach $\mathrm{R}$, Lobstein $\mathrm{T}$. Estimated burden of paediatric obesity and co-morbidities in Europe. Part 1. The increase in the prevalence of child obesity in Europe is itself increasing. Int J Pediatr Obes 2006;1: 26-32.

2. Lobstein T, Jackson-Leach R. Estimated burden of paediatric obesity and comorbidities in Europe. Part 2. Numbers of children with indicators of obesityrelated disease. Int J Pediatr Obes 2006;1:33-41.

3. Buttriss J. Nutrition, health and schoolchildren. Nutr Bull 2002;27:275-316.

4. Ogden CL, Carroll MD, McDowell MA, Flegal KM. Obesity among adults in the United States - no statistically significant change since 2003-2004. NCHS data brief $n^{\circ}$ 1. Hyattsville, MD: National Center for Health Statistics; 2007.

5. Ogden CL, Carroll MD, Curtin LR, McDowell MA, Tabak CJ, Flegal KM. Prevalence of overweight and obesity in the United States, 1999-2004. JAMA 2006;295:1549-55.

6. Daniels SR. Cardiovascular disease risk factors and atherosclerosis in children and adolescents. Cur Atherosclerosis 2001;3:469-85.

7. Maffeis C, Tato L. Long-term effects of childhood obesity on morbidity and mortality. Horm Res 2001;55 Suppl 1:42-5.

8. Dietz WH. Childhood weight affects adult morbidity and mortality. J Nutr 1998;128(Suppl 2):S411-4.

9. Bellizzi MC, Dietz WH. Workshop on childhood obesity: summary of the discussion. Am J Clin Nutr 1999;70:S173-5.
10. Brasil. Ministério da Saúde [homepage on the Internet]. Pesquisa nacional de demografia e saúde da criança e da mulher 2006 [cited 2009 Apr 18]. Available from: http://www.saude.gov.br/pnds2006

11. Carraza FR. Desnutrição energético-protéica. In: Carraza FR, Marcondes E. Nutrição clínica em pediatria. São Paulo: Sarvier; 1991. p. 265-87.

12. CDC (Centers for Disease Control and Prevention). [homepage on the Internet] 2000 CDC Growth charts: United States [Online] Hyaltsville: 2002 [cited 2008 Jun 25]. Available from: http://www.cdc.gov/growthcharts

13. Monteiro CA, Conde WL. Secular trends in malnutrition and obesity among children in S. Paulo city, Brazil (1974-1996). Rev Saude Publica 2000;34(Suppl6): 52-61.

14. Guimarães LV, Barros MB. Differences between the nutritional status of children in public preschools and nutritional transition. J Pediatr (Rio J) 2001;77:381-6.

15. Balaban G, Silva GA. Prevalência de sobrepeso e obesidade em crianças e adolescentes de uma escola da rede privada de Recife. J Pediatr (Rio J) 2001;77:96-100.

16. Leão LS, Araújo LM, Moraes LT, Assis AM. Prevalência de obesidade em escolares de Salvador, Bahia. Arq Bras Endocrinol Metab 2003;47:151-7.

17. Anjos LA, Castro IR, Engstrom EM, Azevedo AM. Growth and nutritional status in a probabilistic sample of schoolchildren from Rio de Janeiro, 1999. Cad Saude Publica 2003;19(Supl 1):S171-9. 
18. Oliveira AM, Cerqueira EM, Oliveira AC. Prevalence of overweight and childhood obesity in Feira de Santana-BA: family detection $\mathrm{x}$ clinical diagnosis. J Pediatr (Rio J) 2003;79:325-8.

19. Costa RF, Cintra IP, Fisberg M. Prevalência de sobrepeso e obesidade em escolares da cidade Santos, SP. Arq Bras Endocrinol Metab 2006;50:60-7.

20. Suñé FR, Dias-da-Costa JS, Olinto MT, Pattussi MP. Prevalence of overweight and obesity and associated factors among schoolchildren in a southern Brazilian city. Cad Saude Publica 2007;23:1361-71.

21. IBGE [homepage on the Internet]. Sistema de informação IBGE - cidades@. [cited 2009 Dec 11]. Available from: http://www.ibge.gov.br/cidadesat/ topwindow.htm?1

22. PNUD [homepage on the Internet]. Programa das Nações Unidas para o Desenvolvimento [cited 2009 Dec 11]. Available from: http://www.pnud.org. br/atlas/tabelas/index.php

23. SEADE. Secretaria de Economia e Planejamento do Estado de São Paulo [homepage on the internet]. Fundação Sistema Estadual de Análise de Dados. Perfil municipal [cited 2009 Dec 11]. Available from: http://www.seade.gov.br/ produtos/perfil/perfil.php

24. De Meira SI. Planejamento e gestão urbanos em Sorocaba-SP: Análise das políticas públicas de habitação popular [tese de mestrado]. Curitiba (PR): UFPR; 2005.

25. Benette DL. O mapa da fome. Jornal Cruzeiro do Sul 2003 Fev 25. Suplemento especial.

26. Ministério da Educação [homepage on the Internet]. Instituto Nacional de Estudos e Pesquisas Educacionais - INEP. Censo Educacional 2007 [cited 2008 Sep 11]. Available from: http://www.ibge.gov.br/cidadesat/topwindow. $\mathrm{htm}$

27. Vincent WJ. Statistics in kinesiology. $2^{\text {nd }}$ ed. Champaign: Human Kinects; 1999.

28. Monteiro CA. Coleta e análise da altura dos alunos ingressantes nas escolas de primeiro grau do país: uma proposta para um sistema nacional de acompanhamento da população. J Ped (Rio J) 1989;65:89-92.

29. Wang Y, Zhang Q. Are American children and adolescents of low socioeconomic status at increased risk of obesity? Changes in the association between overweight and family income between 1971 and 2002. Am J Clin Nutr 2006;84:707-16.

30. de Marins VM, Almeida RM, Pereira RA, Barros MB. Overweight and risk of overweight in schoolchildren in the city of Rio de Janeiro, Brazil: prevalence and characteristics. Ann Trop Paediatr 2002;22:137-44. 